\title{
A Method for Conversational Signal-to-Noise Ratio Estimation in Real-World Sound Scenarios
}

Mansour, Naim; Marschall, Marton; May, Tobias; Westermann, Adam; Dau, Torsten

Published in:

Acoustical Society of America. Journal

Link to article, DOI:

$10.1121 / 1.5101769$

Publication date:

2019

Document Version

Publisher's PDF, also known as Version of record

Link back to DTU Orbit

Citation (APA):

Mansour, N., Marschall, M., May, T., Westermann, A., \& Dau, T. (2019). A Method for Conversational Signal-toNoise Ratio Estimation in Real-World Sound Scenarios. Acoustical Society of America. Journal, 145, 1873. https://doi.org/10.1121/1.5101769

\section{General rights}

Copyright and moral rights for the publications made accessible in the public portal are retained by the authors and/or other copyright owners and it is a condition of accessing publications that users recognise and abide by the legal requirements associated with these rights.

- Users may download and print one copy of any publication from the public portal for the purpose of private study or research.

- You may not further distribute the material or use it for any profit-making activity or commercial gain

- You may freely distribute the URL identifying the publication in the public portal 
 \\ A Method for Conversational Signal-to-noise Ratio Estimation in Real-World Sound Scenarios}

\section{Introduction}

- Analysis of conversational signal-to-noise ratios (SNRs) measured in real-world scenarios can provide insights into communicative strategies and difficulties, and guide development of hearing devices [1].

- Measuring SNRs accurately and realistically is challenging in typical recording conditions, where only a mixture of sound sources is captured. Typical single-channel methods [2] rely on subtracting estimates of noise in a frame $\mathbf{N}_{\mathbf{r}}(\mathbf{f})$ of the recording from the mixture of speech and noise $(\mathbf{S}(\mathbf{f})+\mathbf{N}(\mathbf{f}))_{\mathbf{r}}(1)$.

$$
\operatorname{SNR}(f)=\frac{(S(f)+N(f))_{r}-N_{r}(f)}{N_{r}(f)} \quad(1) \quad S N R(f)=\frac{S_{r}(f)}{N_{r}(f)}
$$

- A novel in-situ estimation method is proposed, where the speech signal of a person in natural conversation is captured by a cheek-mounted microphone, free-field adjusted, and then convolved with a measured impulse response to estimate the clean speech receiver component $\mathbf{S}_{\mathbf{r}}(\mathbf{f})(2)$

- The method is analyzed using in-situ recordings of a real-world workspace meeting and compared to the single-channel technique in terms of its resulting SNR distribution.

\section{Method}

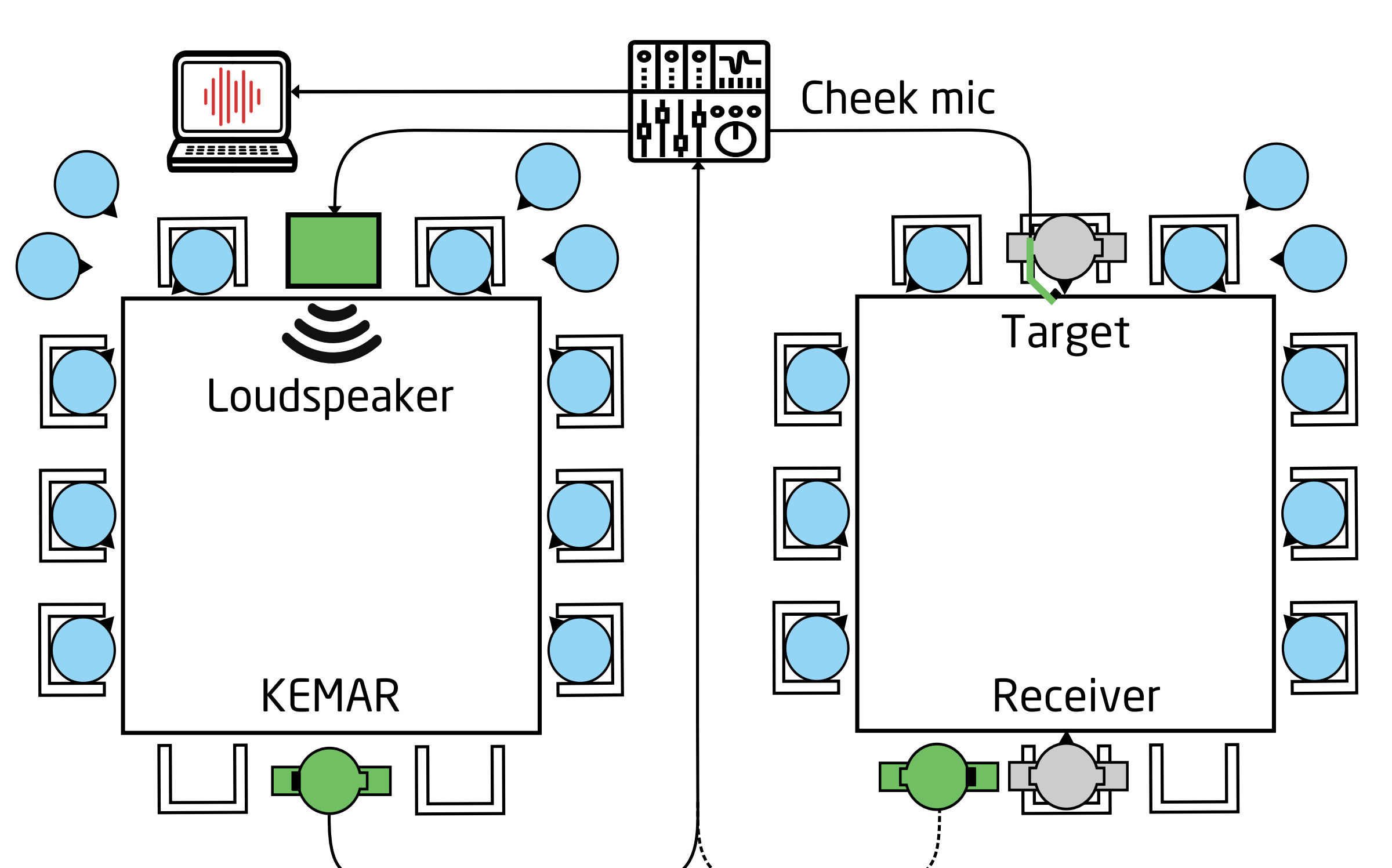

Figure 1. Left: Room impulse response recording - Right: Conversation recording (top-down view)

\section{Controlled recording of workspace meeting}

- Realistic enactment of a typical workspace meeting in a corporate office meeting room

- Conversation between two normal-hearing $(\mathrm{NH})$ people seated across a square conference table, in a background (BG) of $10 \mathrm{NH}$ talkers conversing in pairs about work-related topics

- Room impulse response (RIR) between target and receiver position recorded by KEMAR [3] manikin (left ear), while BG talkers were quiet

- Cheek microphone (CM) captures source speech, KEMAR (right ear) noise at receive SNR estimation

\section{$\rightarrow \rightarrow \begin{gathered}\text { Target speaker } \\ \text { segment removal }\end{gathered}$}
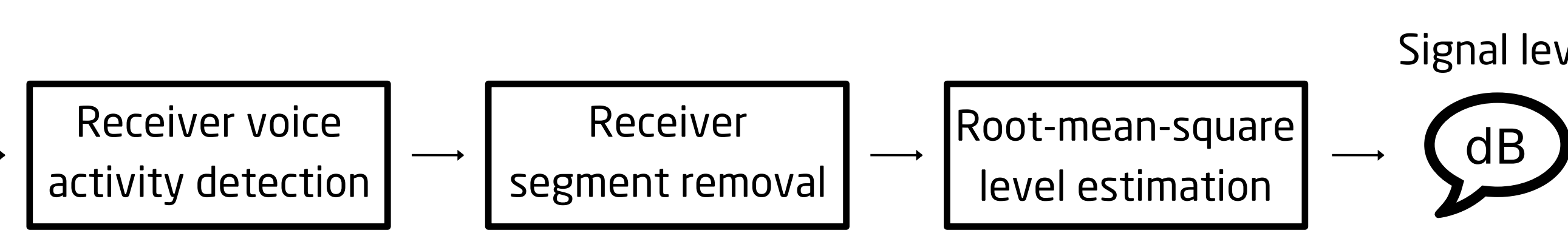

\section{$3, \begin{gathered}\text { Target voice act. } \\ \text { detection }[4]\end{gathered}$}
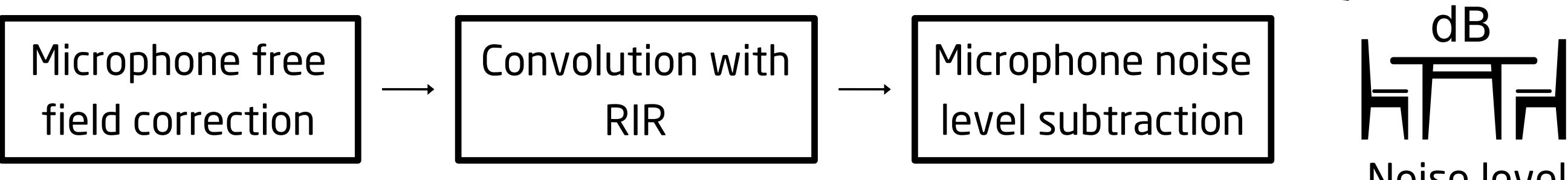

- Frame-based processing, with 10s frame length, and 90\% overlap between frames

- Broad-band SNR based on root-mean-square (RMS) power within each frame

Voice activity detection (VAD) $\boldsymbol{6}$ segment removal

- Energetic VAD [4] to separate speech from background in CM \& KEMAR

- Target speaker segment removal in KEMAR recording through binary mask from CM VAD

Microphone free-field correction

- Transfer function between CM and reference microphone to obtain

free-field acoustical conditions in the target speech signal

- Recorded in anechoic chamber with KEMAR producing white noise at a level of $90 \mathrm{~dB}$, and reference microphone at a distance of $0.5 \mathrm{~m}$

Convolution with room impulse response

- CM signal convolved with appropriately scaled RIR recorded between target \& receiver

Microphone noise level subtraction

- VAD-driven CM RMS level reduction, subtracting BG

noise power in frame nRMS(f) from signal SRMS(f)

$$
S_{c}(f)=s(f) \frac{S_{R M S}(f)-n_{R M S}(f)}{S_{R M S}(f)}
$$

\section{Results}

Free-field correction \& room impulse response

- IR computed from 15 s exponential frequency sweep, FFC low-pass filtered at $10 \mathrm{kHz}$

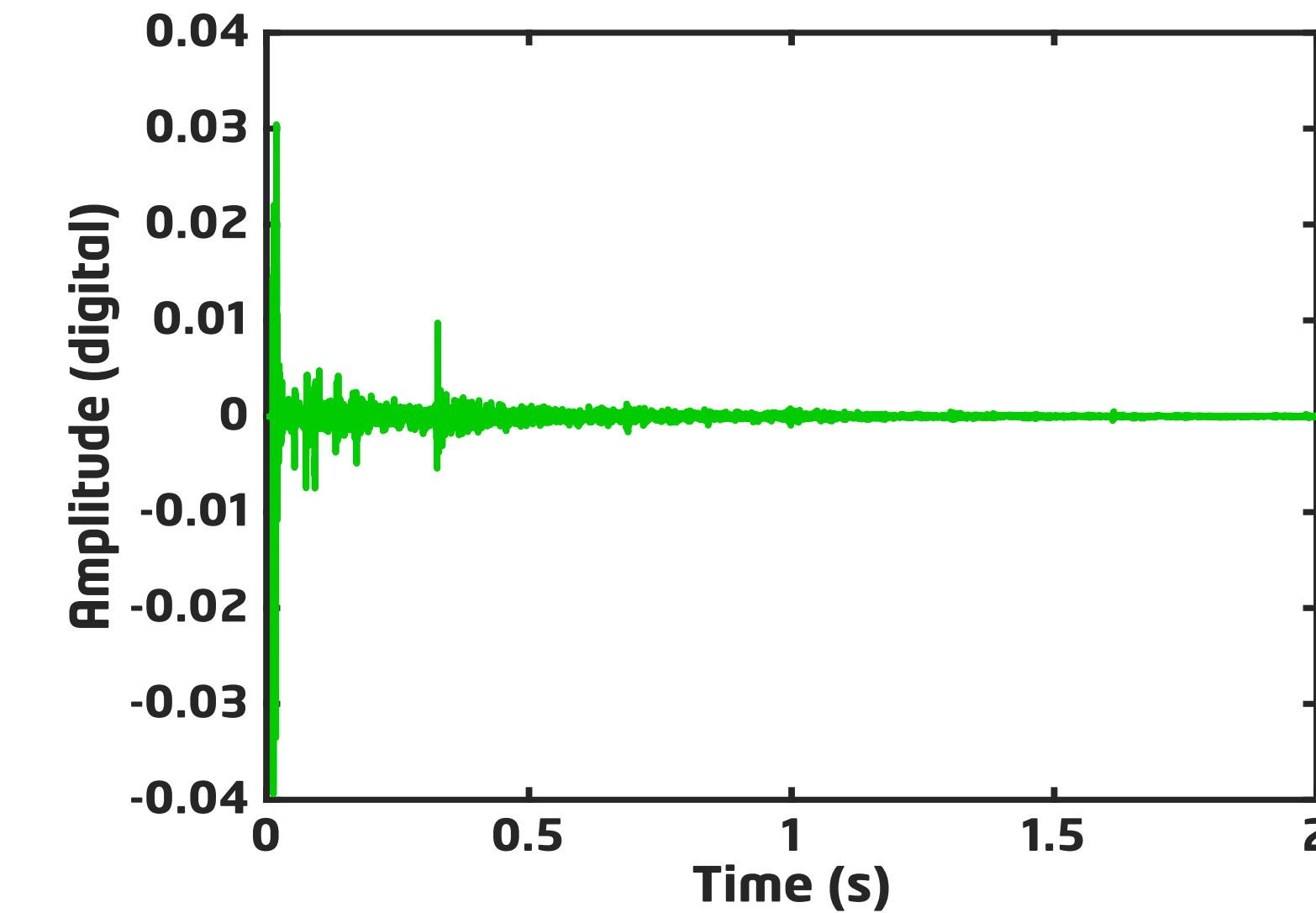

Figure 3. Impulse response

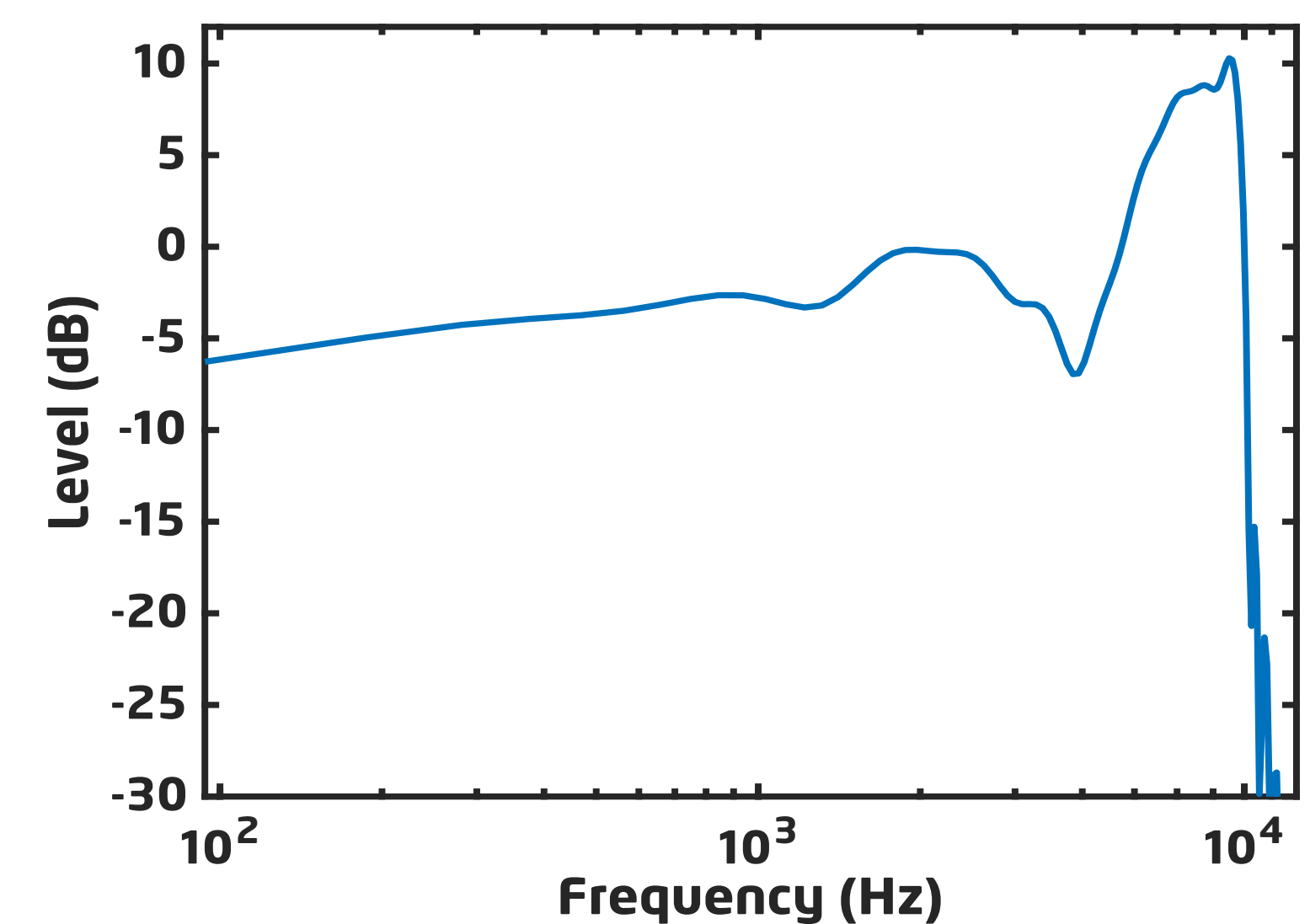

Figure 4. Free-field correction
Speech and background levels

- Derived from 6-minute recording, temporal progression and level distributions are shown

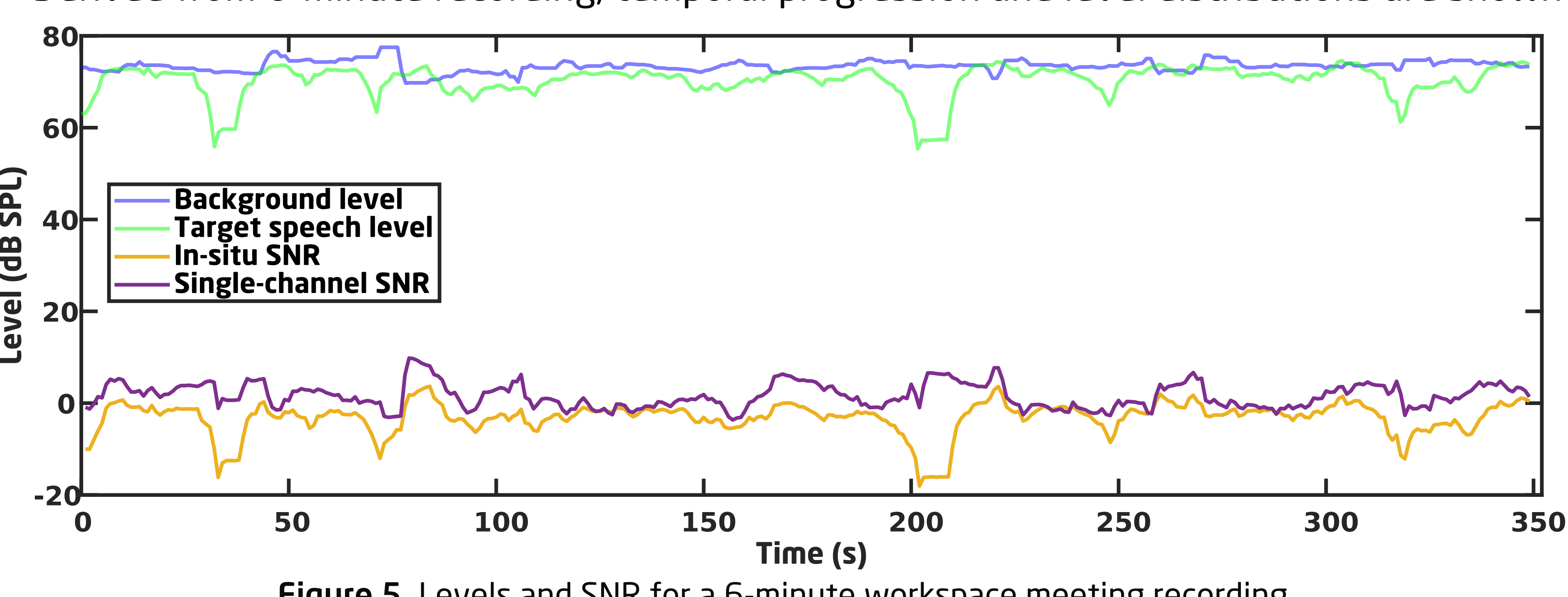

Figure 5. Levels and SNR for a 6-minute workspace meeting recording

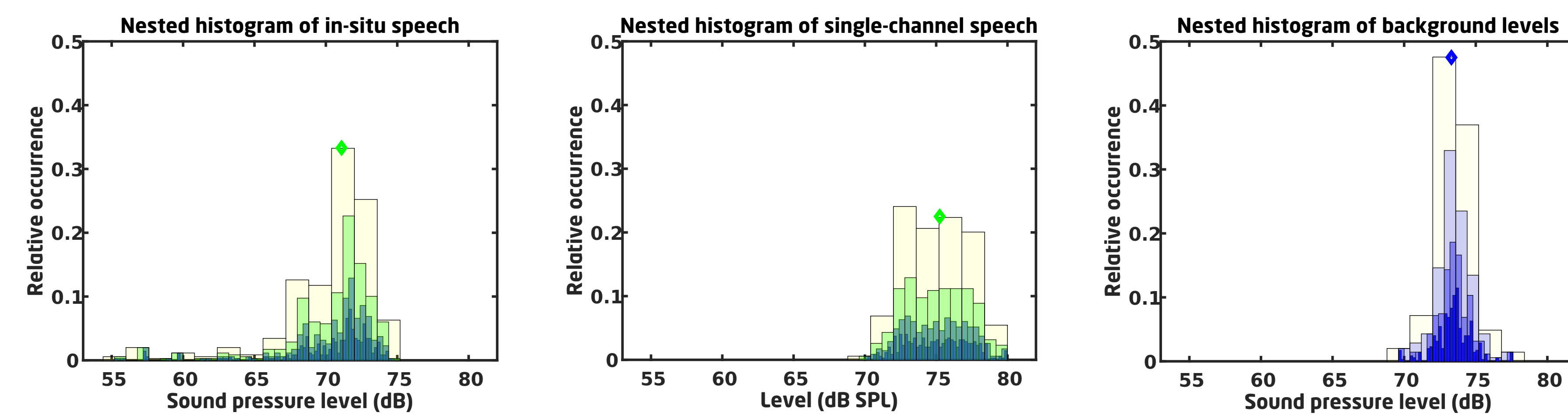

Figure 6. Nested level histograms of in-situ speech, single-channel speech and background levels

Signal-to-noise ratios

- Computed according to respective in-situ (Eqn. 2) and single-channel (1) SNR equations
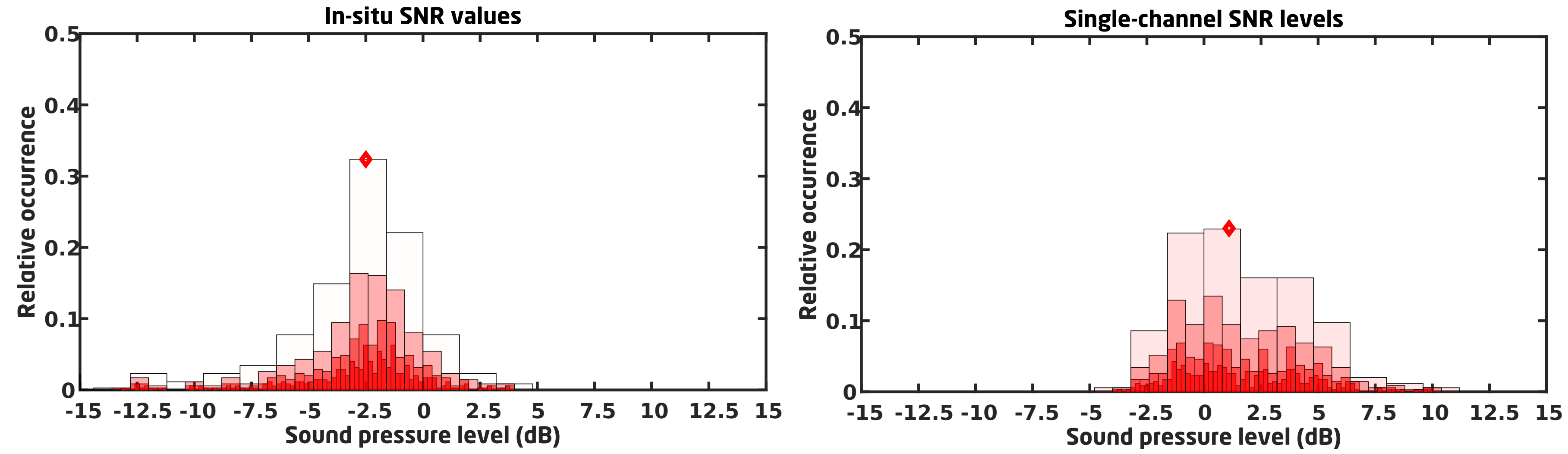

Figure 7. Nested histograms of SNR for in-situ (left) and single-channel (right) method

- Time-averaged dynamic range:

In-situ speech: 55-74 dB Single-channel speech: 70-80 dB Background: 70-76 dB

- Median values, both methods:

In-situ speech: 71 dB $\quad$ Single-channel speech: 75.2 dB Background: 73.5 dB In-situ SNR: - $2.5 \mathrm{~dB}$

\section{Discussion \& Summary}

- A high temporal-resolution measurement technique for speech and background levels allows tracking of in-situ conversational SNR, even at negative values.

- The wide dynamic range found for in-situ speech levels is likely due to natural speech pauses and turn-taking during conversation.

The obtained in-situ SNRs are lower than the corresponding single-channel SNRs, likely due to speech signal being more accurately tracked.

The $4.2 \mathrm{~dB}$ difference between the median in-situ and single-channel SNR indicates a potential overestimation of SNRs with traditional techniques. This is likely due to level effects and correlations between the speech and noise when both are present.

- The in-situ approach requires the availability of a CM signal and suitable RIR recordings.

The proposed SNR estimation method can accurately characterize in-situ SNRs. This may contribute tounderstanding how humans communicate in challenging environments, and help improving compensation strategies in hearing instruments.

\section{References}

[1] Weisser A, Buchholz JM. Conversational speech levels and signal-to-noise ratios in realistic acoustic conditions. The Journal of the Acoustical Society of America 145(1); Jan 2019. p. 349

[2] Smeds K, Wolters F, Rung M. Estimation of signal-to-noise ratios in realistic sound scenarios. The Journal of the American Academy of Audiology 26(2); February 2015. p. 183-196

[3] GRAS Sound \& Vibration, 45BC Knowles Electronic Manikin for Acoustic Research. Head \& Torso with Mouth Simulator, ANSI: S3.36, S3.25, IEC: 60318-7, January 2013

[4] Kinnunen T, Lib H. An overview of text -independent speaker recognition: From features to supervectors. Speech Communication, 52(1);2010. p. 12-40 